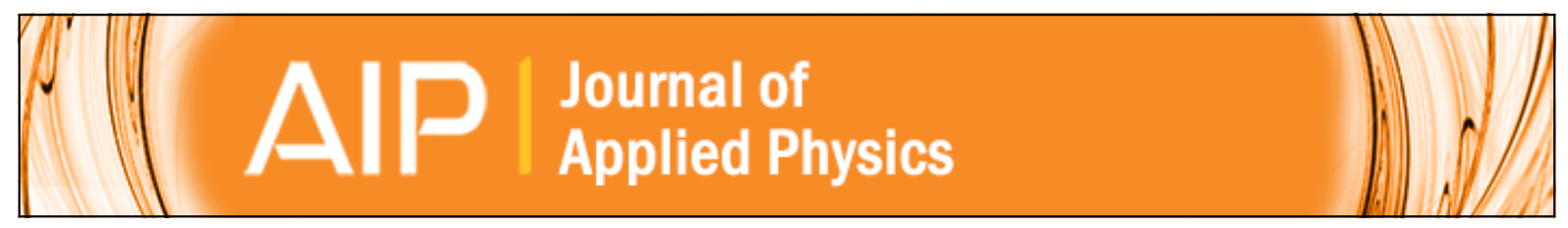

\title{
Transport modeling in ionomeric polymer transducers and its relationship to electromechanical coupling
}

Thomas Wallmersperger, Donald J. Leo, and Curt S. Kothera

Citation: Journal of Applied Physics 101, 024912 (2007); doi: 10.1063/1.2409362

View online: http://dx.doi.org/10.1063/1.2409362

View Table of Contents: http://scitation.aip.org/content/aip/journal/jap/101/2?ver=pdfcov

Published by the AIP Publishing

\section{Articles you may be interested in}

Thickness dependence of curvature, strain, and response time in ionic electroactive polymer actuators fabricated via layer-by-layer assembly

J. Appl. Phys. 109, 104301 (2011); 10.1063/1.3590166

Visualization of the cation migration in ionic polymer-metal composite under an electric field

Appl. Phys. Lett. 96, 043301 (2010); 10.1063/1.3293290

Efficient single-component light-emitting electrochemical cells with an ion-conducting water-soluble polyfluorene Appl. Phys. Lett. 94, 243305 (2009); 10.1063/1.3153511

Wrinkled polypyrrole electrode for electroactive polymer actuators

J. Appl. Phys. 92, 4631 (2002); 10.1063/1.1505674

Ionochromism in a light-emitting electrochemical cell with low response time based on an ionic conductive polyphenylene vinylene

Appl. Phys. Lett. 75, 2014 (1999); 10.1063/1.124900

\section{MIT LINCOLN} LABORATORY CAREERS

Discover the satisfaction of innovation and service to the nation
- Space Control

- Air \& Missile Defense

- Communications Systems \& Cyber Security

- Intelligence, Surveillance and

Reconnaissance Systems

$$
\begin{aligned}
& \text { - Advanced } \\
& \text { Electronics } \\
& \text { - Tactical Systems } \\
& \text { - Homeland } \\
& \text { Protection } \\
& \text { - Air Traffic Control }
\end{aligned}
$$

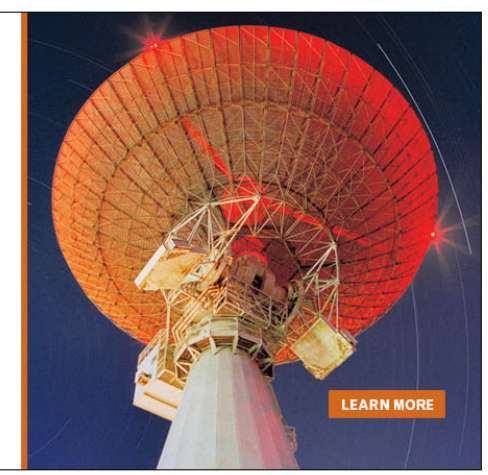




\title{
Transport modeling in ionomeric polymer transducers and its relationship to electromechanical coupling
}

\author{
Thomas Wallmersperger ${ }^{a)}$ \\ Institut für Statik und Dynamik der Luft-und Raumfahrtkonstruktionen, Universität Stuttgart, \\ Pfaffenwaldring 27, D-70569 Stuttgart, Germany \\ Donald J. Leo ${ }^{\text {b) }}$ and Curt S. Kothera \\ Center for Intelligent Material Systems and Structures, Department of Mechanical Engineering, \\ Virginia Tech, Blacksburg, Virginia 24061
}

(Received 29 July 2006; accepted 2 November 2006; published online 25 January 2007)

\begin{abstract}
Ionomeric polymer transducers consist of an ion-conducting membrane sandwiched between two metal electrodes. Application of a low voltage $(<5 \mathrm{~V})$ to the polymer produces relatively large bending deformation ( $>2 \%$ strain) due to the transport of ionic species within the polymer matrix. A computational model of transport and electromechanical transduction is developed for ionomeric polymer transducers. The transport model is based upon a coupled chemoelectrical multifield formulation and computes the spatiotemporal volumetric charge density profile to an applied potential at the boundaries. The current induced in the polymer is computed using the isothermal transient ionic current associated with surface charge accumulation at the electrodes induced by nonzero volumetric charge density within the polymer. The stress induced in the polymer is assumed to be a summation of linear and quadratic functions of the volumetric charge density. Euler-Bernoulli beam mechanics are used to compute the bending deflection of the transducer to an applied potential. The diffusion coefficient and permittivity of the polymer is identified from the measured current density to a step change in the applied potential. A comparison between the measured data and the predicted response demonstrates that this model accurately predicts the current discharge due to the applied potential at voltages over the range of $50-500 \mathrm{mV}$. Furthermore, the measured strain response is accurately predicted by determining the two parameters of the mechanics model that relates volumetric charge density to induced stress. The coupled model with parameters identified from the step response analysis is used to predict the harmonic response of the current and the bending strain. Comparisons between measured data and simulations illustrate that the coupled transport-mechanics model accurately predicts the magnitude and trends associated with the current response and strain output. Excellent agreement is obtained at excitation periods above approximately $1 \mathrm{~s}$ while good agreement is obtained at longer excitation periods. The transport model highlights the importance of the asymmetry that develops at large applied potentials and long excitation periods in the volumetric charge density due to the fixed anionic species in the polymer. (C) 2007 American Institute of Physics. [DOI: 10.1063/1.2409362]
\end{abstract}

\section{INTRODUCTION}

Ionomeric polymer transducers, also called ionic polymer-metal composites, are transducers that exhibit electromechanical sensing and actuation properties. It is well established that ionomer materials, when suitably plated with conductive metal on their surfaces, exhibit a bending deformation when a voltage is applied across the thickness. ${ }^{1}$ Conversely, the materials exhibit a sensing response under the application of a mechanical deformation. ${ }^{2,3}$ These properties allow them to be used as electromechanical sensors and actuators. In comparison with other types of materials that exhibit electromechanical coupling, such as piezoelectric materials, ionomeric materials have been shown to produce bending strains on the order of $2 \%$ under the application of potentials on the order of $1-5 \mathrm{~V}$. Recent advances in the development of "dry" materials have also enabled the use of

\footnotetext{
${ }^{a)}$ Electronic mail: wallmers@isd.uni-stuttgart.de

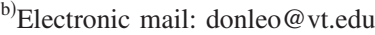

these ionomeric materials in air, ${ }^{4,5}$ thus increasing the practicality of using these materials in environments that do not require control over hydration or humidity.

Although a number of advances have been made in the development of ionomeric transducers in recent years, there still remains a gap in understanding the fundamental mechanisms that promote electromechanical coupling. It has been well established for a number of years that ion conduction in the polymer due to the existence of mobile charged species accounts for the existence of electromechanical coupling. Beyond that, though, there have been a number of models based on different physical phenomena that can closely match experimental data. The models that provide a direct comparison between experiment and theory can generally be separated into phenomenological models based on curvefits of experimental data ${ }^{6-9}$ and physics-based models that attempt to predict the material response from governing equations.

The purpose of this paper is to develop a computational 


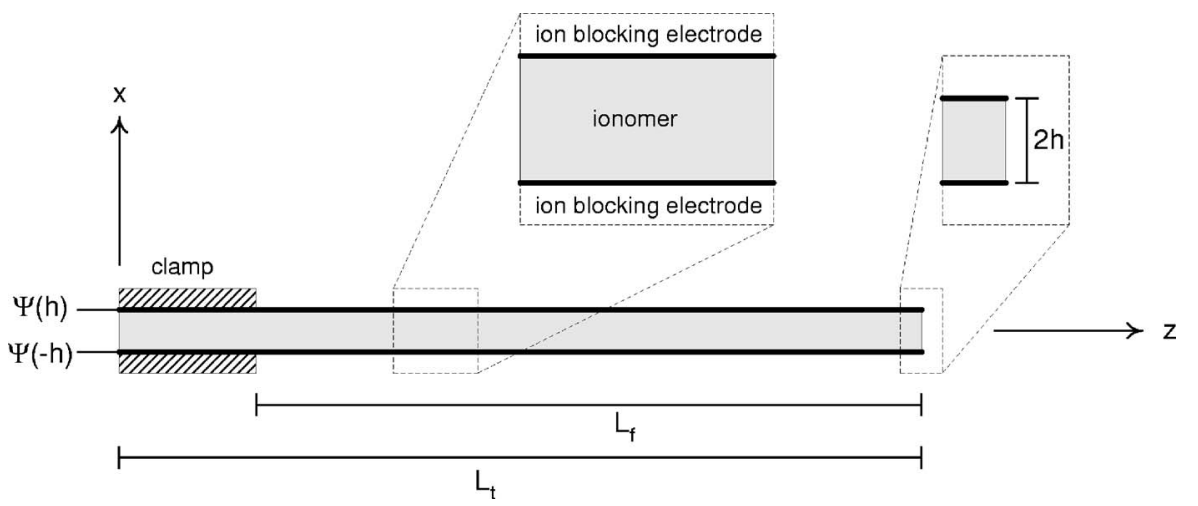

FIG. 1. Transducer geometry for the computational and experimental analysis.

methodology that solves for the electromechanical response of ionomeric transducers. The computational framework that we develop in this paper is based on the solution of the one-dimensional Nernst-Planck equations for charge conduction considering the effects of concentration gradient and electric field. The computational methodology computes the spatiotemporal charge density as a function of applied field and boundary conditions. The computational framework is able to account for the existence of both mobile and immobile charge species in the polymer. The mechanical response of the polymer is computed by assuming a series relationship between charge density and local stress. Euler-Bernoulli beam mechanics are then applied to compute the bending moment and deflection of the polymer as a function of time and applied field.

The benefit of the computational framework developed in this paper is that it can be readily extended to multidimensional analysis and to materials with more complicated properties. For instance, there is mounting evidence that the interface between the metal electrode and the ionomeric polymer plays a critical role in the electromechanical transduction. ${ }^{10}$ Modeling the spatially dependent diffusion properties and geometric properties of the polymer-metal interface is very difficult with a closed-form analysis, but it is a straightforward extension of the computational framework developed in this paper. Similarly, modeling the effects of ion blocking boundary conditions or boundary conditions with nonzero flux terms can be accomodated within the present framework, whereas it would be difficult to easily model these effects with a closed-form solution.

In this paper we present the one-dimensional transport model and couple it to the mechanics of beam deflection. Simulation results for the current response and bending response of the transducer are compared to experimental data to determine the validity of the model assumptions and determine if experimental trends can be accurately predicted with the computational model.

\section{COMPUTATIONAL MODEL OF ION TRANSPORT}

The system under analysis in this work is a bending beam transducer fabricated from an ionomer substrate with conductive metal electrodes on the outer surfaces. The beam is assumed to be clamped at one end and free at the other. The total length of the beam is denoted $L_{t}$ and the free length is denoted $L_{f}$, as shown in Fig. 1. The thickness of the beam is $2 h$ and the total width is $w$. For the purposes of this analysis we assume that the only nonzero charge transport is in the $x$ direction and the only significant bending occurs along the $z$ axis. The potential is denoted $\Psi(x)$ and is applied at the electrodes. It is assumed that the resistance of the electrodes in the $z$ direction is negligible, therefore the applied potential is equivalent at any point along the length of the beam. The electrodes are assumed to be ion blocking electrodes, meaning that the charged species cannot cross the boundary at $x$ $= \pm h$. Furthermore we assume that there are no electrochemical reactions at the electrodes.

A coupled chemoelectrical formulation describing the ion distribution and the electric potential in the material is applied $^{11,12}$ to compute the charge density as a function of space and time.

\section{A. Chemical field}

The formulation for the chemical field is based on the balance equation for the flux of the mobile ions and fixed charges. Neglecting the convective flux-due to an applied (convective) velocity of the solvent-the one-dimensional Nernst-Planck-Equation is given by

$$
J_{\alpha x}(x, t)=-\underbrace{D_{\alpha} c_{\alpha, x}(x, t)}_{\text {diffusive flux }}-\underbrace{\frac{F}{R T} z_{\alpha} D_{\alpha} c_{\alpha}(x, t) \Psi_{, x}(x, t)}_{\text {migration flux }}
$$

where $J_{\alpha x}(x, t)$ is the flux for each species $\alpha$ in the $x$ direction.

Using the conservation of mass (while neglecting the source terms),

$$
\dot{c}_{\alpha}(x, t)=-J_{\alpha x, x}(x, t),
$$

and Eq. (1), the convection-diffusion equation for each species $\alpha$,

$$
\dot{c}_{\alpha}(x, t)=\left[D_{\alpha} c_{\alpha, x}(x, t)+z_{\alpha} c_{\alpha}(x, t) \mu_{\alpha} \Psi_{, x}(x, t)\right]_{, x},
$$

is obtained. The variable $c_{\alpha}$ is the concentration of the species $\alpha, D_{\alpha}$ the diffusion constant, $\mu_{\alpha}=(F / R T) D_{\alpha}$ is the unsigned mobility, $z_{\alpha}$ the valence of the ions, and $\Psi$ is the electric potential. The notation ${ }_{, x}=\partial / \partial x$ denotes a partial derivative with respect to $x$.

\section{B. Electrical field}

The electric field is described by the one-dimensional Poisson equation, 


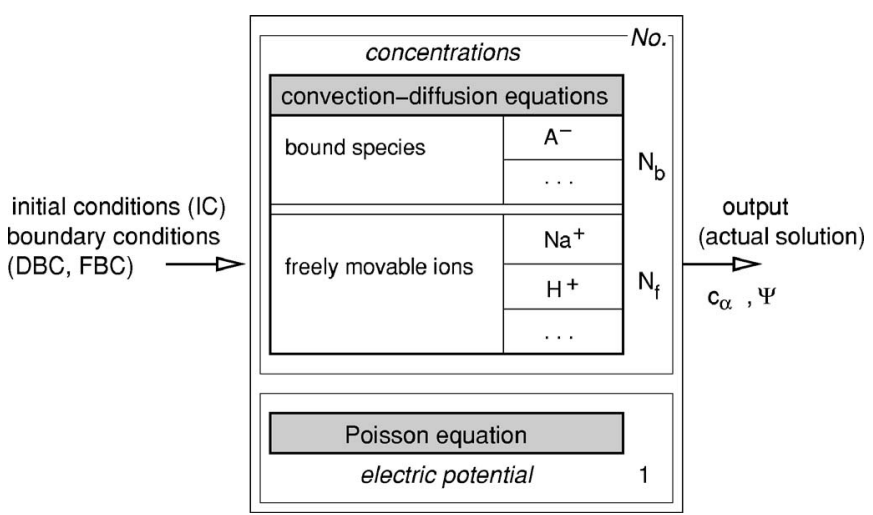

FIG. 2. Chemoelectric coupling scheme.

$$
\Psi_{, x x}(x, t)=-\frac{F}{\varepsilon \varepsilon_{0}} \sum_{\alpha=1}^{N_{f}+N_{b}}\left[z_{\alpha} c_{\alpha}(x, t)\right] .
$$

Due to the much larger propagation speed in the electric field compared to the diffusion speed in the chemical field, the quasistatic form of the Poisson equation is sufficient. Equation (4) is based on the second and fourth Maxwell equations,

$$
E_{x}=-\Psi_{, x}, \quad D_{x, x}=\rho,
$$

where $\varepsilon_{0}$ is the permittivity of free space, $\varepsilon$ the relative dielectric constant, $e$ the electric elementary charge, $F$ the Faraday constant $\left(F=N_{A} e=96487 \mathrm{C} / \mathrm{mol}\right), D_{x}$ the electric displacement vector, $E_{x}$ the vector of the electric field strength, and $\rho$ the density of volume charge. $N_{f}$ and $N_{b}$ denote the number of freely movable species and the number of bound species, respectively.

\section{Coupling of the chemoelectric field}

The chemoelectric multifield consists of two coupled partial diferential equations:

- the convection-diffusion equations, Eq. (3), which are partial differential equations (PDEs) of first order in time for the concentrations $c_{\alpha}$ of all the species $\alpha$, and

- the Poisson equation, Eq. (4), a PDE in space for the electric potential $\Psi$.

A coupling method, in which the convection-diffusion equations for all $N_{f}+N_{b}$ species and the Poisson equation for the electric field are solved simultaneously, has been considered, see scheme of the coupling method in Fig. 2. This results in a total number of nodal unknowns in space of $N_{f}$ $+N_{b}+1$, for more details see Ref. 13. For the computations in this work only one mobile species and one fixed charge is employed.

\section{Boundary conditions}

Boundary conditions (BCs) for the electrical field and chemical field must be specified to solve the governing equations. For the electric field we prescribe the electric potential at both domain boundaries as fixed values [Dirichlet boundary conditions (DBC)]. For the chemical field different approaches are possible.
- First, we can apply at both domain boundaries fixed values for the concentration.

- A second possibility is to apply prescribed fluxes over the domain boundaries [Flux boundary conditions (FBC)]; in this case for each species a distinct flux $\bar{J}_{\alpha}$ may be prescribed as

$-D_{\alpha} c_{\alpha, x}-\frac{F}{R T} z_{\alpha} D_{\alpha} c_{\alpha} \Psi=\bar{J}_{\alpha x}$.

- The third possibility is to apply combined BCs: at one boundary a DBC can be applied, and at the other one a FBC.

In this work we have specified a zero flux boundary condition at $x= \pm h$ to model the ion blocking electrode at each surface.

\section{E. Electric current}

The electric current density $j_{x}$ can be determined by the Faraday equation,

$j_{x}(x, t)=F \sum_{\alpha=1}^{N_{f}}\left\{z_{\alpha}\left[-D_{\alpha} \frac{\partial c_{\alpha}}{\partial x}(x, t)-z_{\alpha} \mu_{\alpha} c_{\alpha}(x, t) \frac{\partial \Psi}{\partial x}(x, t)\right]\right\}$.

The calculation of $j_{x}$ may be done in a postprocessing step using the nodal values and the local gradients in the whole domain.

\section{F. Current density computation}

The numerical simulation of ion transport in the polymer produces the spatiotemporal distribution of charge density and potential. The charge density is defined as

$$
\rho(x, t)=F \sum_{\alpha=1}^{N_{f}+N_{b}}\left[z_{\alpha} c_{\alpha}(x, t)\right] .
$$

The distribution of charge density within the polymer gives rise to image charges which collect at the faces of the electrodes. In the absence of ion flux across the boundary, the electrode forms an ion blocking boundary and the image charges that accumulate at the surface induce the current that can be measured across the thickness of the polymer. This effect has been denoted as the isothermal transient ionic current, or ITIC, by Ref. 14. The surface charge per unit area, $q^{s}(t)$, induced by the existence of a nonzero charge density $\rho(x, t)$ within the sample is

$$
q^{s}(t)=\frac{1}{2 h} \int_{-h}^{h} x \rho(x, t) d x .
$$

The resulting current per unit area is the time derivative of the induced surface charge,

$$
i(t)=\frac{d q^{s}(t)}{d t} .
$$

The surface charge and current are computed by Eqs. (9) and (10), respectively, using the discretized solution of the transport analysis. The integration in Eq. (9) is performed using a 
trapezoidal numerical integration and the differential of the surface charge is computed using a first-order approximation for the time derivative.

\section{G. Electromechanical transduction}

The electromechanical transduction models studied in this paper focus on the relationship between charge density and localized stress within the polymer. Physics-based models of local stresses using a micromechanics approach have derived a set of relationships between the charge density and the mechanical stress and strain generated. ${ }^{15}$

The transduction model proposed in this paper is an expansion of the form

$$
\sigma(x, t)=\alpha \rho(x, t)+\beta \rho^{2}(x, t),
$$

where $\alpha$ and $\beta$ are coefficients that define the linear and quadratic terms of the electromechanical transduction model. The bending moment produced in the beam due to this stress distribution is

$$
\begin{aligned}
M(t) & =\int_{-w / 2}^{w / 2} \int_{-h}^{h} x \sigma(x, t) d x d y \\
& =\int_{-w / 2}^{w / 2} \int_{-h}^{h} x\left[\alpha \rho(x, t)+\beta \rho^{2}(x, t)\right] d x d y .
\end{aligned}
$$

To analyze the effect of the linear and quadratic terms on the response, we decompose the bending moment into two terms,

$$
\begin{aligned}
& M_{\alpha}(t)=\alpha\left[w \int_{-h}^{h} x \rho(x, t) d x\right], \\
& M_{\beta}(t)=\beta\left[w \int_{-h}^{h} x \rho^{2}(x, t) d x\right],
\end{aligned}
$$

and rewrite $M(t)=M_{\alpha}(t)+M_{\beta}(t)$. Assuming that the EulerBernoulli beam theory applies to the material, the expression for the curvature is

$$
\mathcal{K}(t)=\frac{M(t)}{E I},
$$

where $E I$ is the effective bending stiffness of the beam. The strain at any point in the beam can be written as

$$
\epsilon(t)=-x \mathcal{K}(t),
$$

and the maximum strain is equal to $h \mathcal{K}(t)$ at the outer fibers of the transducer.

\section{COMPUTATIONAL METHODS}

The coupled multifield problem has been solved on an adaptive multigrid with variable discretization to find the optimal mesh, i.e., maximal accuracy of the numerical results for a minimal computational effort. The refinement of the mesh $g$ is defined by the ratio of the lengths $l$ of two subsequent elements $k$ and $k+1$,

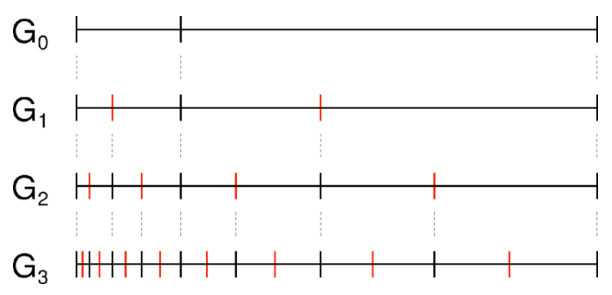

FIG. 3. (Color online) Adaptive refinement of a four-level multigrid where $g(0)=4$.

$$
g=\frac{l_{k+1}}{l_{k}}>1 .
$$

By using a geometric series, the length $\Delta x_{\min }$ of the first element (which is the smallest element) may be calculated by

$$
\begin{aligned}
& \Delta x_{\min }\left(1+g+g^{2}+g^{3}+\cdots+g^{n}\right) \\
& =\Delta x_{\min } \sum_{k=0}^{n} g^{k}=\Delta x_{\min } \frac{g^{n+1}-1}{g-1}=l_{x},
\end{aligned}
$$

where $l_{x}=2 h$ is the domain length and $n_{\mathrm{el}}=n+1$ is the number of elements. To obtain a consistent refinement, a different refinement factor $g$ (level) has to be chosen for all multigrid levels,

$$
g(\text { level })=[g(0)]^{0.5^{\text {level }}} \text { where level }=0,1,2, \ldots .
$$

In Fig. 3 a multigrid consisting of four levels-adaptively refined where $g(0)=4$-is depicted.

To find the optimal mesh geometry, the following meshes $G_{0}$ are investigated:

- equidistant meshes: meshes with constant element lengths $(g=1)$ with $50,100,200,400, \ldots$ elements and

- meshes with refinement factors $g(0)=1.05$ and $g(0)$ $=1.10$ with $50,100,200,400, \ldots$ elements.

As an example, a mesh with 100 elements divided in three subdomains with a refinement factor of $g(0)=1.1$ on both sides of each subdomain is given in Fig. 4.

In Fig. 5 the normalized mobile concentrations $c / c^{-}$ $=c / c_{\text {fix }}$ over the number of total elements on the finest mesh at the position $x=-h$ [Fig. 5(a)] and $x=+h$ [Fig. 5(b)] are plotted for the different refinement factors. The convergence for the different mesh geometries, i.e., the error of the normalized concentration $\epsilon=\left|c-c_{\text {exact }}\right| / c_{\text {exact }}$ over the number of total elements $n_{\mathrm{el}}$, at $x=-h$ is given in Fig. 6(a) and at $x$ $=+h$ in Fig. 6(b).

As we can see in Fig. 5, the results of the concentrations of the different discretizations converge to approximately the same value for both the concentrations at the top $(x=+h)$ and the ones at the bottom $(x=-h)$. The convergence is quadratic

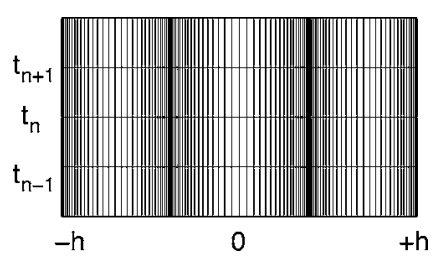

FIG. 4. Adaptively refined mesh comprising three subdomains. 


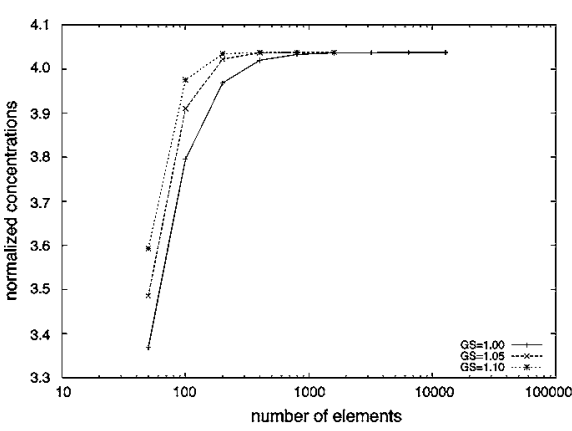

(a)

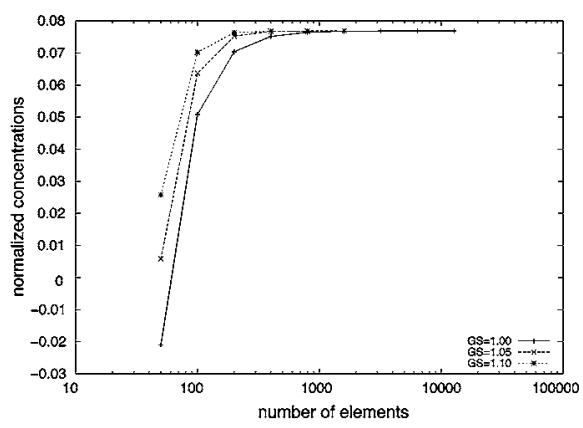

(b) $\left[\varepsilon \sim(\Delta \bar{l})^{2}\right]$ for $g=1$ and of higher order for the adaptively refined geometries, see Fig. $6 . \bar{l}$ is the mean value of the element lengths, $\bar{l}=2 h / n_{\mathrm{el}}$.

Note, for the refined geometries the ratio of $r$ $=\Delta x_{\max } / \Delta x_{\min }$ is increasing for large numbers of elements, see Table I. This large ratio causes numerical difficulties and thus the error $\varepsilon$ is not falling below a certain limit.

For a prescribed error of $\varepsilon \leqslant 0.1 \%$, we have chosen a discretization on the finest mesh of 400 elements and a refinement factor $g(0)=1.05$ to have a minimal computational effort and also a not too high ratio $r$. These mesh parameters have been used for all of the remaining analyses presented in this work.

\section{EXPERIMENTAL PROCEDURES}

This section will present experimental results to validate the model development that was discussed previously. The experimental methods and laboratory setup will be discussed first, followed by the measured results.

\section{Experimental Setup}

The cantilever polymer actuator used in these experiments had a total length of $29 \mathrm{~mm}$, a width of $3 \mathrm{~mm}$, and a thickness of approximately $0.5 \mathrm{~mm}$. Being a cantilever sample, $9 \mathrm{~mm}$ of the material was placed in the clamp, leaving a free length of $20 \mathrm{~mm}$. This polymer sample was prepared using a direct assembly process as outlined in Ref. 16. The particular makeup of this actuator includes Nafion ${ }^{\circledR}-117$ as the base ionomer, lithium as the cation, and nearly 100 wt \% uptake of formamide for the solvent. Each of the two electrodes is $25 \mu \mathrm{m}$ thick and is composed of $30 \mathrm{vol} \%$ $\mathrm{RuO}_{2}$ and a thin layer of gold foil. The direct assembly pro- cess enables higher performance actuators with the possibilities of incorporating large solvent uptakes.

Experiments were performed with this sample using the test setup shown in Fig. 7. A dSPACE DS2103 12 bit digitalto-analog converter $( \pm 5 \mathrm{~V})$ supplied the voltage wave form, which passed through a Hewlett-Packard 6825A bipolar supply/amplifier to amplify the current before reaching the polymer. Voltage and current measurements were collected at this point, with the help of a current sensing circuit made from an operational amplifier and two resistors. This signal then excited the polymer sample. The clamp that holds the cantilever actuator is made of Delrin ${ }^{\circledR}$ and applies the voltage to the polymer through gold foil electrodes. A Polytec OFV 3001 laser vibrometer controller and 303 sensor head were used to measure the mechanical response of the polymer actuator, with the measurement point taken in the middle of the beam, $10 \mathrm{~mm}$ from the base. All the data were collected using a dSPACE DS2003 16 bit analog-to-digital converter set to $\pm 5 \mathrm{~V}$. In each set of experiments, two electrical signals (voltage and current) and two mechanical signals (displacement and velocity) were measured. Also included in Fig. 7 are arrows to show the flow of these signals and the polymer dimensions of free length $L_{f}$, width $w$, and total thickness $2 h$.

Two sets of experiments were conducted on the cantilever actuator. The first set were step responses testing six different voltage levels: 50, 100, 250, 500, 750, and $1000 \mathrm{mV}$. Three averages were taken at each voltage to assure repeatable results. The sample rate here was set to its maximum of $10 \mathrm{kHz}$ to get the closest estimation possible of the peak current. Incidentally, a set of tests was run with the sample rate at $1 \mathrm{kHz}$ and they showed peak currents at nearly half those measured at $10 \mathrm{kHz}$. For the second set of experiments, single-tone sine wave responses were measured at

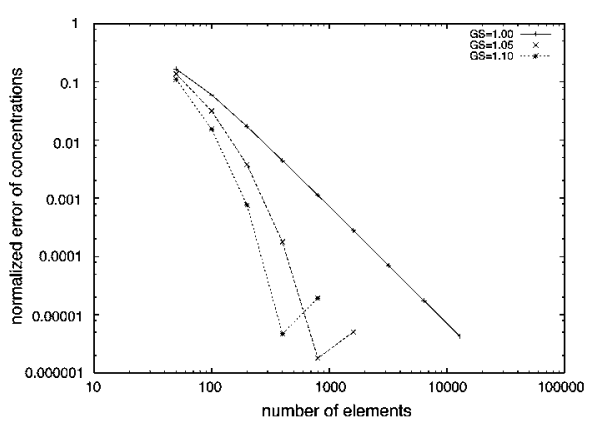

(a)

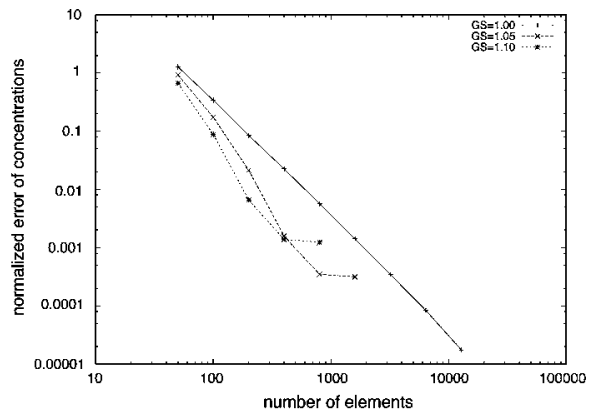

(b)
FIG. 6. Error of the normalized concentration $\varepsilon=\left|c-c_{\text {exact }}\right| / c_{\text {exact }}$ over the number of total elements $n_{\mathrm{el}}$ at (a) $x=$ $-h$ and (b) $x=+h$. 
TABLE I. Ratio of largest to smallest element length for different numbers of elements; refinement factor $g(0)=1.1$.

\begin{tabular}{lccccc}
\hline \hline No. of elements $n_{\mathrm{el}}$ & 50 & 100 & 400 & 800 & 1600 \\
$r=\Delta x_{\max } / \Delta x_{\min }$ & $>2$ & $>4$ & $>400$ & $>10^{5}$ & $>10^{10}$ \\
\hline
\end{tabular}

various frequency and amplitude settings. The frequency range spanned four decades from 0.01 up to $100 \mathrm{~Hz}$ at halfdecade increments, giving nine total frequencies, with four different voltage amplitudes tested at each: 50, 100, 500, and $1000 \mathrm{mV}$. The sample rate for the sine experiments was adjusted at each frequency to give a constant number of data points per cycle, and in order to maximize the available rate, this number was set to 100 .

\section{STEP RESPONSE ANALYSIS}

Step response measurements are used to determine the diffusion coefficient and permittivity associated with the equations for charge transport due to an applied electric potential. The diffusion coefficient and permittivity are chosen by plotting the peak current density and the charge density of the each data set for the measured response. The charge density of the measured response is computed by integrating the measured current density over the time period of $0-10 \mathrm{~s}$. The peak current density and charge density are computed using simulations of the charge density response using Eqs. (9) and (10). The diffusion coefficient and permittivity are then chosen to match the simulated response to the measured data over the voltage range of $50-500 \mathrm{mV}$. The results of this analysis are shown in Figs. 8(a) and 8(b). The results demonstrate that the simulations are able to accurately predict the peak current density and the total charge density. The parameters used in the simulation and the resulting diffusion coefficient and permittivity are shown in Table II.

Determination of the diffusion coefficient and permittivity allows us to analyze the spatiotemporal charge density profile predicted by the simulation. At an applied step potential of $50 \mathrm{mV}$, the steady-state charge density profile is approximately symmetric through the thickness of the polymer, as shown in Fig. 9(a). Increasing the amplitude of the step change produces a charge density profile that exhibits a "depletion region" at the anode due to the fixed anion concentration $c^{-}$. Plotting the normalized charge density results in a region of charge depletion that saturates at -1 . At the opposing electrode (the cathode) the charge accumulates to a value much larger than the initial cation concentration of the

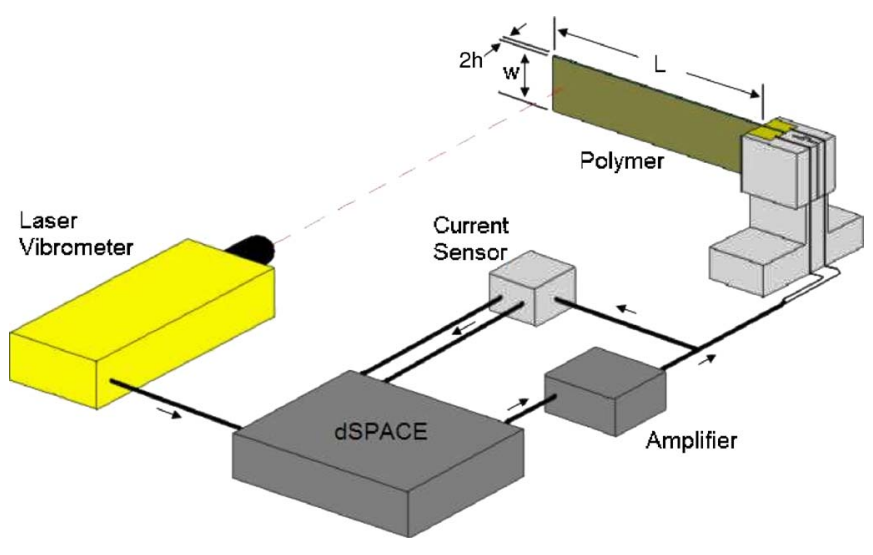

FIG. 7. (Color online) Laboratory setup for cantilever ionic polymer actuator experiments.

polymer. Increasing the voltage amplitude increases the depletion region at the anode and increases the charge accumulation at the cathode. These results are consistent with those of Refs. 14 and 15 for single-ion transport.

The time response of the current density to a step change in potential is computed and compared to the measured data using the parameters determined from the analysis of the peak current and charge density. The results shown in Fig. 9(b) demonstrate that fitting the diffusion coefficient and permittivity of the material produces excellent agreement between the predicted current response to a step change in potential and the measured data. The model accurately predicts the time constant associated with the discharge of current for all four voltage levels tested. One noticeable discrepancy between the measured data and the model is the steady-state current that occurs for the $500 \mathrm{mV}$ step change in potential. The model assumption that the electrode is ion blocking precludes the development of a steady-state current in the simulations.

The strain response to a step change in voltage is also simulated by choosing the parameters that correlate charge density to local stress response. Combining Eqs. (12), (14), and (15) we can write the maximum strain response as

$$
\epsilon_{\max }(t)=-\alpha \frac{w h}{E I} \int_{-h}^{h} x \rho(x, t) d x-\beta \frac{w h}{E I} \int_{-h}^{h} x \rho^{2}(x, t) d x .
$$

Equation (19) can be nondimensionalized by substituting $x$ $=h \xi$ into the expression and rewriting

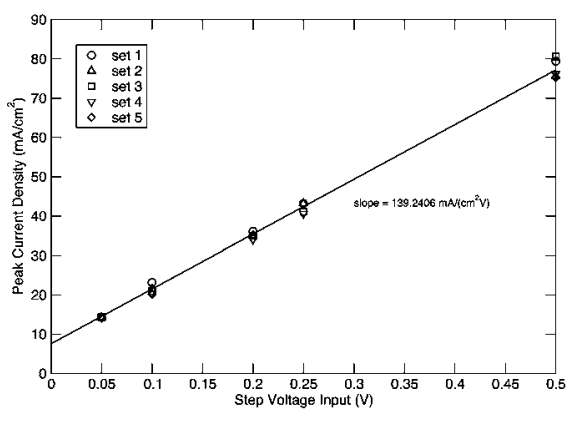

(a)

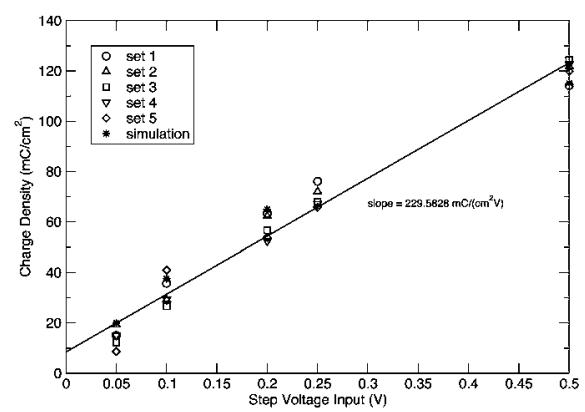

(b)
FIG. 8. (a) Peak current density as a function of voltage and (b) charge density as a function of voltage. 
TABLE II. Simulation parameters.

\begin{tabular}{ccc}
\hline \hline Parameter & Variable & Value \\
\hline Polymer thickness & $2 h$ & $180 \mu \mathrm{m}$ \\
Anion concentration & $c^{-}$ & $1073 \mathrm{~mol} / \mathrm{m}^{3}$ \\
Diffusion coefficient & $D_{\alpha}$ & $6 \times 10^{-11} \mathrm{~m}^{2} / \mathrm{s}$ \\
Dielectric constant & $\varepsilon \varepsilon_{0}$ & $17.7 \mathrm{mF} / \mathrm{m}$ \\
Temperature & $T$ & $293 \mathrm{~K}$ \\
& $\hat{\alpha}$ & 8600 \\
& $\hat{\beta}$ & 850 \\
\hline \hline
\end{tabular}

$\epsilon_{\max }(t)=-\alpha \frac{w h^{3} c^{-}}{E I} \int_{-1}^{1} \xi \hat{\rho}(\xi, t) d \xi-\beta \frac{w h^{2} c^{-2}}{E I} \int_{-1}^{1} \xi \hat{\rho}^{2}(\xi, t) d \xi$,

where $\hat{\rho}=\rho / c^{-}$. The terms in the integrals are computed from the simulated spatiotemporal charge density profile. To eliminate the need to measure the geometric and elastic properties of the transducer, we define

$$
\begin{aligned}
& \hat{\alpha}=\alpha \frac{w h^{3} c^{-}}{E I}, \\
& \hat{\beta}=\beta \frac{w h^{2} c^{-2}}{E I}, \\
& \hat{\epsilon}_{\text {lin }}(t)=\int_{-1}^{1} \xi \hat{\rho}(\xi, t) d \xi, \\
& \hat{\epsilon}_{\text {quad }}(t)=\int_{-1}^{1} \xi \hat{\rho}^{2}(\xi, t) d \xi .
\end{aligned}
$$

Substituting these definitions into Eq. (20) produces the relationship

$$
\epsilon_{\max }(t)=-\hat{\alpha} \hat{\epsilon}_{\text {lin }}(t)-\hat{\beta} \hat{\epsilon}_{\text {quad }}(t) .
$$

Defining the strain relationship in terms of the assumed material parameters $\hat{\alpha}$ and $\hat{\beta}$ and the charge density profile allows us to analyze the relative importance of the linear and quadratic terms in the strain response of the material. Recalling Fig. 9 we note that the charge density profile is approximately symmetric at low applied potentials and becomes increasingly asymmetric at higher potentials due to the depletion of the anode caused by the immobility of the anions. The terms $\hat{\epsilon}_{\text {lin }}(t)$ and $\hat{\epsilon}_{\text {quad }}(t)$ are computed for the steady-state charge density profiles for applied voltages of 50, 100, 200, and $500 \mathrm{mV}$. The results shown in Fig. 10(a) illustrate that $\hat{\epsilon}_{\text {lin }}(t) \gg \hat{\epsilon}_{\text {quad }}(t)$ for voltage amplitude on the order of $50 \mathrm{mV}$, but that $\hat{\epsilon}_{\text {quad }}(t) \gg \hat{\epsilon}_{\text {lin }}(t)$ when the potential increases to $500 \mathrm{mV}$. This is consistent with the fact that $\hat{\epsilon}_{\text {quad }}(t)$ would be equal to zero if the charge density profile was perfectly symmetric about the middle of the beam.

The relationship between the linear and quadratic strain term is used to determine the two-parameter model for the strain response. The parameter $\hat{\alpha}$ is chosen to match the strain response at the smallest potential $(50 \mathrm{mV})$ due to the fact that the charge density is nearly symmetric at this voltage level, thus minimizing the effect of the quadratic term in the stress-to-charge density model. The parameter $\hat{\beta}$ is then chosen to fit the strain response at the higher voltage levels. The resulting parameters are listed in Table II.

The comparison between the simulated strain response and the measurements illustrates that the two-parameter model is able to accurately fit the data. Figure 10(b) demonstrates that the choice of parameters produces a model that fits the measured data over the potential range of 50-500 $\mathrm{mV}$. The time constant of the step response in strain is also accurately predicted, supporting the model assumption that the local stress is correlated with the charge density profile that arises due to the step change in potential applied to the material. Also shown in Fig. 10(b) is the predicted response for a model in which $\hat{\beta}=0$. The results demonstrate the importance of the quadratic term in predicting the strain response. Although a linear model would be able to fit any one of the four measurements, it is clear that choosing the one-parameter model, i.e., $\hat{\alpha} \neq 0, \hat{\beta}=0$, would not be able to accurately predict the measured step response at multiple values of potential. In contrast, a two-parameter model is able to accurately represent the data over a range of potential values and the measurements support the assumption that the quadratic term in the two-parameter model will become more prominent as the voltage increases.

\section{HARMONIC RESPONSE ANALYSIS}

The transport model and mechanics model were also correlated to the measured harmonic response of the transducer. The comparisons were performed over the frequency
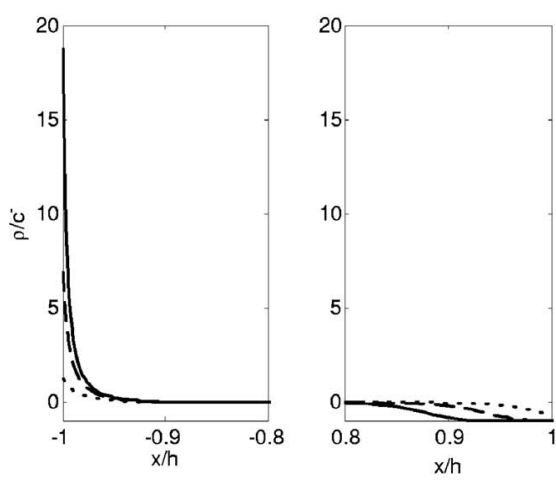

(a)

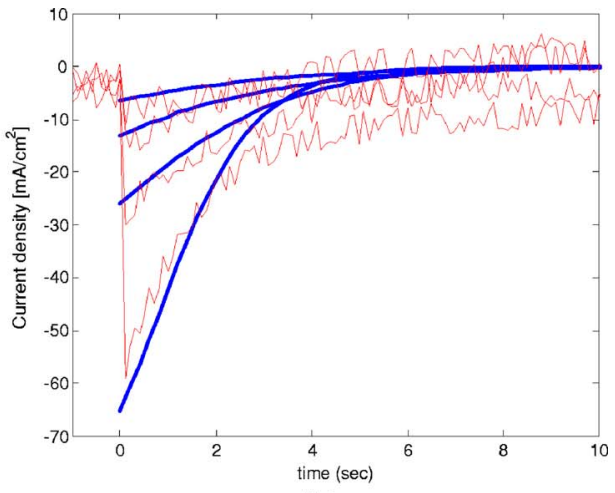

(b)
FIG. 9. (Color online) (a) Spatiotemporal charge density profile predicted by the simulation for a step change in the applied voltage: $500 \mathrm{mV}$ (solid), $200 \mathrm{mV}$ (dashed), and $50 \mathrm{mV}$ (dotted). (b) Current response to a step change in potential: model (solid) and measured (dashed) for 50, 100, 200, and $500 \mathrm{mV}$ 


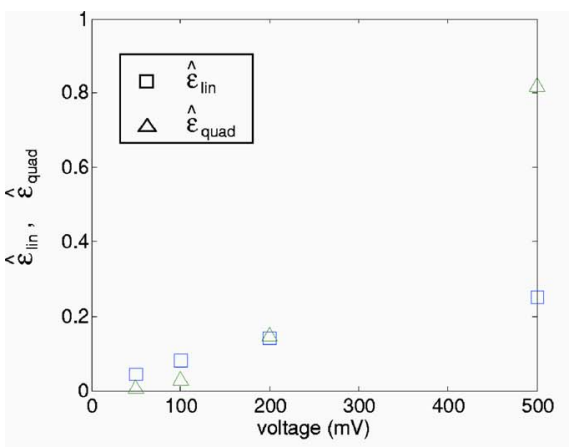

(a)

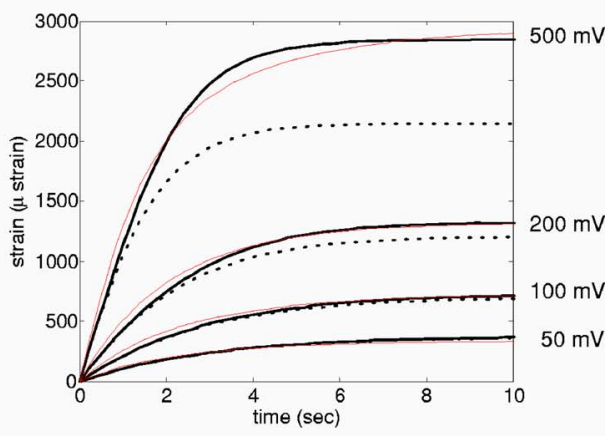

(b) range of $0.1-10 \mathrm{~Hz}$ using the model parameters determined from the step response correlation. These parameters are listed in Table II.

The volumetric charge density was computed as a function of time for the applied boundary potential

$$
\Psi(h)=A \cos \left(\frac{2 \pi}{T} t\right), \quad \Psi(-h)=0,
$$

where $A$ is the amplitude of the applied harmonic excitation and $T$ is the period. An important feature of the temporal response of the volumetric charge density is the saturation that occurs at large voltage and large excitation periods. For large enough applied potentials and large periods, a depletion region appears on one of the electrodes due to the motion of mobile charges. This result is shown in Fig. 11(a) for the case of $A=500 \mathrm{mV}$ and $T=10 \mathrm{~s}$. The large accumulation of charge on one electrode causes full depletion of mobile charges on the opposite electrode, resulting in a normalized volumetric charge density that saturates at -1 . Thus, the asymmetry that is computed for step changes in potential also occurs in the harmonic response at sufficiently high applied potentials and sufficiently long excitation periods.

Simulations also demonstrate that the asymmetry in the volumetric charge density does not occur at sufficiently short excitation periods. Simulations for the case of $A=500 \mathrm{mV}$ and $T=0.1 \mathrm{~s}$ illustrate that the volumetric charge density is symmetric at each instant of time when the excitation period is decreased to $0.1 \mathrm{~s}$, see Fig. 11(b). For the diffusion and permittivity parameters identified for this model, the transition from asymmetric to symmetric profiles occurs at an excitation period between 1 and $2 \mathrm{~s}$.
The peak-to-peak current density simulated by the model was compared to measured values for two values of potential amplitude. The frequency response of the peak-to-peak current density is compared for 100 and $500 \mathrm{mV}$ in Fig. 12(a). The simulation accurately predicts the magnitude of the current density as well as the trends with frequency. The accuracy of the model is lowest at frequencies of approximately $0.1 \mathrm{~Hz}$ for the $500 \mathrm{mV}$ amplitude, although the accuracy increases at higher frequencies. The lower accuracy at high electric voltages and at low frequencies may be attributed to the fact that the model does not include the actual electrode architecture (geometry and surface area). Therefore it is believed that the model is overemphasizing the asymmetry of the charge.

The time response of the maximium strain in the transducer is also computed using the values of the two-parameter mechanical model identified from the step response analysis. Comparing the predicted peak-to-peak strain with the measured values for two amplitudes of the applied potential (100 and $500 \mathrm{mV}$ ) illustrates that the same parameters identified by the step response analysis can accurately predict the magnitude as well as the trends associated with decreasing the excitation period, see Fig. 12(b). The model predicts a decreasing strain response over the frequency range of $0.1-10 \mathrm{~Hz}(T=10 \mathrm{~s}$ to $T=0.1 \mathrm{~s})$ and this prediction accurately represents the trends in the measured data. Furthermore, the accuracy of the model increases at higher frequencies. We attribute this increase in accuracy to the reduced effect of the quadratic term in the strain response of the transducer. As discussed in the previous section, a nearly

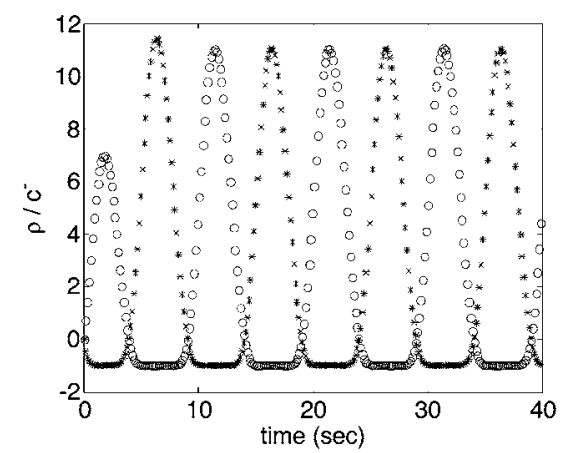

(a)

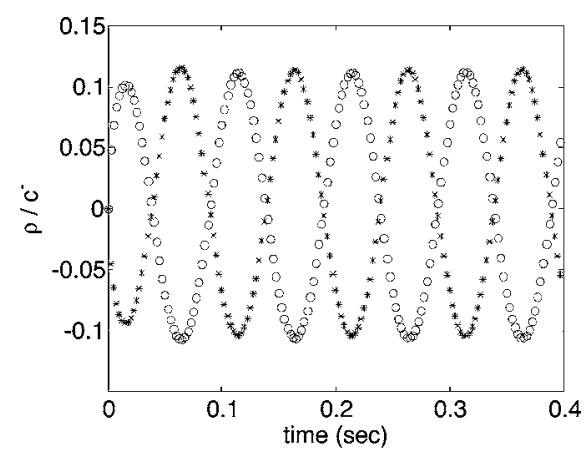

(b)
FIG. 11. Normalized charge density on each of the electrodes for a harmonic excitation: (a) amplitude equals $500 \mathrm{mV}$ and period equals $10 \mathrm{~s}$ and (b) amplitude equals $500 \mathrm{mV}$ and period equals $0.1 \mathrm{~s}$. 


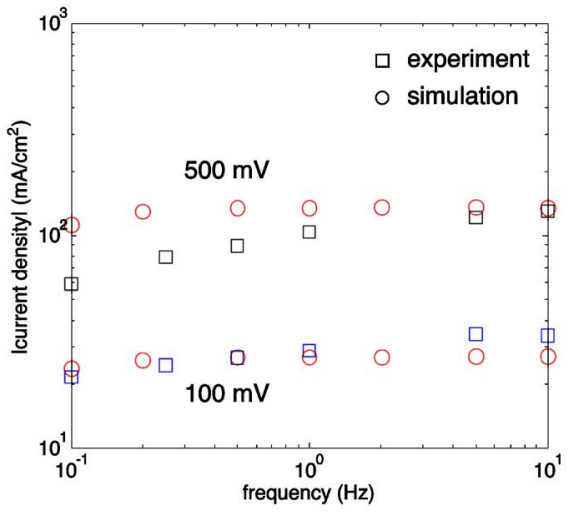

(a)

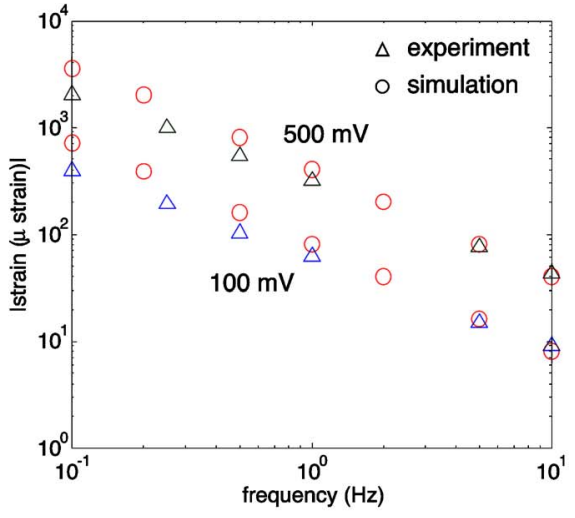

(b)
FIG. 12. (Color online) (a) Frequency response of current density for harmonic excitation: experimental investigations $(\square)$ and numerical simulation (O). (b) Frequency response of strain for harmonic excitation: experiment $(\triangle)$ and simulation $(\bigcirc)$ symmetric volumetric charge density profile results in a mechanical strain response that is dominated by the linear term in Eq. (22).

\section{SUMMARY AND CONCLUSIONS}

The combination of a one-dimensional transport model with a parametric model of the beam response produces a predictive model of the electromechanical response of ionomeric polymer transducers. The one-dimensional model is parametrized by the diffusion coefficient and the permittivity of the polymer, which, in turn, determine the peak response and time constant of the current due to an applied step voltage. Determining these two parameters from measured data produces a model that accurately predicts the current discharge over a wide range of voltage levels. The model assumption that the stress induced by charge transport is related to the integral of the charge density and charge density squared accurately predicts the strain response of the polymer for step changes in applied voltage. The amplitude of the strain response as a function of frequency is also accurately predicted over two decades in frequency $(0.1-10 \mathrm{~Hz})$. In addition to accurately predicting the amplitude, the decrease in strain response for increasing frequency is also predicted, supporting the use of the two-parameter model that relates induced stress-to-charge density.

\section{ACKNOWLEDGMENTS}

Portions of this work were supported by the National Science Foundation CAREER Award under Contract/Grant No. CMS-0093889.

${ }^{1}$ K. Oguro, Y. Kawami, and H. Takenaka, J. Micromachine Soc. 5, 27 (1992).

${ }^{2}$ K. Sadeghipour, R. Salomon, and S. Neogi, Smart Mater. Struct. 1, 172 (1992).

${ }^{3}$ M. Shahinpoor, Y. Bar-Cohen, J. Simpson, and J. Smith, Smart Mater. Struct. 7, R15 (1998).

${ }^{4}$ M. Shahinpoor and K. Kim, Appl. Phys. Lett. 80, 3445 (2002).

${ }^{5}$ M. Bennett and D. J. Leo, Sens. Actuators, A 115, 79 (2004).

${ }^{6}$ R. Kanno, S. Tadokoro, T. Takamori, and M. Hattori, Proceedings of the 1996 IEEE International Conference on Robotics and Automation, Minneapolis, Minnesota, April 1996, pp. 219-225.

${ }^{7}$ K. M. Newbury and D. J. Leo, J. Intell. Mater. Syst. Struct. 14, 333 (2003).

${ }^{8}$ K. M. Newbury and D. J. Leo, J. Intell. Mater. Syst. Struct. 14, 343 (2003).

${ }^{9}$ S. Nemat-Nasser, S. Zamani, and Y. Tor, J. Appl. Phys. 99, 104902 (2006).

${ }^{10}$ B. Akle, M. Bennett, and D. Leo, Sens. Actuators, A 126, 173 (2006).

${ }^{11}$ T. Wallmersperger, B. Kröplin, and R. W. Gülch, Mech. Mater. 36, 411 (2004).

${ }^{12}$ T. Wallmersperger, B. Kröplin, and R. W. Gülch, Electroactive Polymer (EAP) Actuators as Artificial Muscles; Reality, Potential, and Challenges; 2nd ed. (SPIE, Bellingham, WA, 2004), Vol. PM136, pp. 335-362.

${ }^{13}$ T. Wallmersperger, Ph.D. thesis, Universität Stuttgart, 2003.

${ }^{14}$ G. Frenning and M. Stromme, J. Appl. Phys. 90, 5570 (2001).

${ }^{15}$ S. Nemat-Nasser, J. Appl. Phys. 92, 2899 (2002).

${ }^{16}$ B. Akle, M. Bennett, and D. Leo, Proc. SPIE 5759, 153 (2005). 